\title{
Acute exacerbation of idiopathic pulmonary fibrosis
}

\section{To the Editor:}

We read with interest the recent paper by AMBRosinI et al. [1], reporting five patients with acute exacerbation of idiopathic pulmonary fibrosis (IPF). As confirmed by this detailed clinicopathological study, acute exacerbation of IPF is generally characterised histologically by areas of diffuse alveolar damage superimposed on a background of usual interstitial pneumonia and is associated with a poor shortterm prognosis. The possibility that it may be sustained by foci of organising pneumonia instead of diffuse alveolar damage, although briefly pointed out in the literature [2-5], has never been evaluated in any detail.

Recently, we encountered a 77-yr-old male, former smoker (30 packs $\cdot \mathrm{yr}^{-1}$ ), presenting with fever, dry cough and progressive dyspnoea lasting $1 \mathrm{yr}$. No signs of infection, congestive heart failure or collagen vascular disease were present, and no history of drug toxicity or environmental exposure was reported. A chest radiograph and a subsequent computed tomography (CT) scan showed a parenchymal opacification in the right upper lobe, and bilateral, perpipheral reticular opacities with traction bronchiolectasis and honeycombing in the lower lobes. The patient was treated with antibiotics and steroids, and symptoms improved and the opacification of the right upper lobe disappeared. After the rapid tapering of steroids due to side-effects, the patient was admitted again with fever and acute exacerbation of dyspnoea. At high-resolution CT, multifocal ground-glass attenuations were present, beside the pre-existing bi-basal reticular opacities and honeycombing. Fibreoptic bronchoscopy with bronchoalveolar lavage was performed, the latter revealing the following profile: cellularity $800,000 \cdot \mathrm{mm}^{-3}$, macrophages $26 \%$, lymphocytes $17 \%$, neutrophils $54 \%$, eosinophils $3 \%$ and CD4:CD8 0.86 . Type II reactive cells were present, whereas pulmonary pathogens were not found. A videothoracoscopic biopsy from the upper and the lower lobes of the left lung was performed. Histologically, in the upper lobe, fibroblastic plugs protruded into the alveolar lumen, with mild interstitial inflammation and a preservation of the pulmonary architecture; in the lower lobe, dense interstitial fibrosis with honeycombing and scattered fibroblastic foci emanated from the subpleural region and alternated with small centrolobular areas of relatively normal lung. The patient was treated with low-dose steroids and azathioprine, which led to an improvement in the symptoms and to the resolution of the ground-glass attenuations. Fifteen months after biopsy, the patient is alive with progressive fibrosis.

This case is characterised by two different histological patterns: organising pneumonia in the upper lobe and usual interstitial pneumonia in the lower lobe. Interestingly, even in the absence of areas of diffuse alveolar damage, it satisfies the current clinical definition of acute exacerbation of IPF [1, 4]. In our opinion, this case prompts the following considerations. 1) Histologically, in some patients with IPF, the acute exacerbation is sustained by an organising pneumonia pattern, instead of the typical diffuse alveolar damage. In other words, some patients with IPF associated with organising pneumonia may have clinico-radiographical findings and enough functional impairment to fulfil the current definition of acute exacerbation of IPF [5]. 2) In patients with IPF, the distinction between those in who an acute exacerbation is sustained by organising pneumonia and those in who it is sustained by diffuse alveolar damage is probably important, because the former may have a significantly better short-term prognosis than the latter. Further investigation on a larger series is needed to verify this hypothesis. 3) As stated by AMBROSINI et al. [1], in the correct clinico-radiological scenario, bronchoalveolar lavage findings may be sufficient for a correct diagnosis of acute exacerbation of IPF, and probably, in some cases, also to differentiate an accelerated phase sustained by organising pneumonia from an accelerated phase sustained by diffuse alveolar damage. However, clinical and radiographical findings are sometimes quite similar in the two situations, and in the most difficult cases the distinction requires a surgical lung biopsy.

As the pathological process in patients with idiopathic pulmonary fibrosis is heterogeneous, it is important to obtain specimens from more than one lobe at the time of biopsy, as in our case.

\author{
R. Dallari*, M. Foglia*, M. Paci ${ }^{\oplus}$, A. Cavazza \\ *Unit of Pulmonology, Ospedale di Sassuolo, Modena, ${ }^{\#}$ Unit \\ of Thoracic Surgery, and "Unit of Pathology, Ospedale \\ S. Maria Nuova, Reggio Emilia, Italy.
}

\section{References}

1. Ambrosini V, Cancellieri A, Chilosi M, et al. Acute exacerbation of idiopathic pulmonary fibrosis: report of a series. Eur Respir J 2003; 22: 821-826.

2. American Thoracic Society/European Respiratory Society international multidisciplinary consensus classification of the idiopathic interstitial pneumonias. Am J Respir Crit Care Med 2002; 165: 277-304.

3. Travis WD, Colby TV, Koss MN, Rosado-de-Christenson ML, Muller NL, King TE. Non neoplastic disorders of the lower respiratory tract. In: West King D, ed. Atlas of Nontumor Pathology. Washington DC, American Registry of Pathology and Armed Forces Institute of Pathology, 2002; pp. 68-69.

4. Akira M, Hamada H, Sakatani M, et al. CT findings during phase of accelerated deterioration in patients with idiopathic pulmonary fibrosis. Am J Roengtenol 1997; 168: 79-83.

5. Nagata N, Nagatomo H, Yoshii C, Nikaido Y, Kido M. Features of idiopathic pulmonary fibrosis with organising pneumonia. Respiration 1997; 64: 331-335. 